\title{
Life Cycle Support for PLC Controlled Manufacturing Systems.
}

\author{
Jacqueline Jarvis ${ }^{a}$ and Dennis Jarvis ${ }^{b}$ \\ ${ }^{a}$ University of South Australia, School of Computer and Information \\ Science, The Levels, Sth. Aust., 5095, Australia. jacquie.jarvis@ \\ unisa.edu.au \\ ${ }^{b}$ CSIRO Division of Manufacturing Technology, P.O. Box 4, Woodville \\ Sth. Aust., 5011, Australia.dhj@adl.dmt.csiro.au
}

\begin{abstract}
Software support for PLC controlled manufacturing systems has focused primarily on the design and production phases of the control system lifecycle - there is limited support for the lifecycle of the manufacturing system itself. Also those phases of the system lifecycle after system development are poorly supported because effective support tools for these phases need to address both the control system and the manufacturing system. We believe that if a model of the total system is constructed at an early stage in the lifecycle which captures both the behaviour of the control system and the behaviour of the manufacturing system then the opportunity exists to develop a suite of model-based tools to support the lifecycle of a particular system from design onwards. We have tested this hypothesis by developing a methodology to enable models of PLC controlled manufacturing systems to be generated efficiently and constructing model-based software to assist in maintenance, diagnosis and system migration activities. We have also identified other activities within the lifecycle that would benefit from such software. The software that we have developed is in support of a $700 \mathrm{i} / \mathrm{o}$ point, 3 station assembly line.
\end{abstract}

\section{Keywords}

programmable logic controllers, maintenance, model-based diagnosis 


\section{INTRODUCTION}

In certain manufacturing sectors, such as the automobile and food processing industries, the quest for automation and flexibility has resulted in extremely complex manufacturing systems, which are typically controlled by Programmable Logic Controllers (PLCs). These systems exhibit a lifecycle similar to that of conventional software systems, which involve the following phases (Birrell and Ould, 1985):

- project inception

- system definition

- system design

- system production

- system acceptance

- maintenance

- obsolescence

Manufacturing systems differ from conventional software systems in that there are two subsystems to consider - the control system and the system being controlled (such as an assembly line). The linkage between the two systems is provided by wiring diagrams which are maintained separately from the PLC program and the pneumatic and hydraulic circuit diagrams which typically constitute the documentation for the system being controlled. This separation makes system maintenance particularly difficult because in order to understand the behaviour of the system one needs to understand how both sub-systems behave and how they interact with each other. This problem is further compounded because the information that one has access to is often inconsistent and incomplete. Inconsistencies often arise because the subsystems and their linkages are maintained using separate systems. Incompleteness occurs because typically not all the information required to understand the behaviour of the system (such as the state behaviours and the initial states of entities in the manufacturing system) is explicitly documented.

Our primary motivation was to develop an effective diagnostic system for an existing PLC controlled assembly line. Diagnostic systems for PLC controlled manufacturing systems have been developed using rule-based and other approaches (Myers and Davis, 1990; Milne et al, 1994; Wheeler and Rosetti, 1993; Day and Rostosky, 1994). However, their deployment in systems of the complexity found in the automotive and food processing industries has been extremely limited, as conventional rule-based approaches are unable to produce comprehensive systems in reasonable time-frames (Cirocco et al, 1995). The underlying reason for this is that rule-based diagnostic systems are constructed by associating a set of states with a set of observed faults. This association is normally performed by people who have had extensive experience with the underlying manufacturing system. Unfortunately, when dealing with complex, one-off manufacturing systems, it becomes impossible for a single person (or even a group of people) to experience a sufficiently wide range of fault states to generate a comprehensive diagnostic system. Furthermore, the knowledge acquisition task involved in the development of such a system is extremely time consuming (and therefore expensive), thus providing more disincentive. 
It is our belief that a model-based approach offers a much more attractive path for the development of comprehensive and timely diagnostic systems. In a model-based approach, one would first construct a model of the manufacturing system. Reasoning strategies would then be developed that enabled an association to be made dynamically between an observed fault state and an underlying cause. As noted above, such a model would need to capture the behaviours of both the control system and the system being controlled. Furthermore, we expect that such models would prove to be extremely useful in other maintenance activities and in other phases of the system lifecycle. We are unaware of any attempts to construct models of this kind, although there has been some interest in representing existing control logic with alternative representations in order to facilitate analysis of the control program. Representations that have been used include AND / OR graphs (Cirocco et al, 1995; Day and Rostosky, 1994), Boolean equations (Moon, 1994; Falcione and Krogh, 1993; Boullart, 1992) and logic networks (Asfahl and Balagamwala, 1990). Wheeler and Rossetti (1993) constructed a semantic network representation of a PLC-controlled spinning line in a nylon yarn factory for diagnostic purposes. The semantic network acted as a repository for functional, behavioural, structural and heuristic knowledge relating to the line. Of particular interest to us is that the behavioural knowledge was generated automatically from the PLC programs. However, the outcome of this process was not a dynamic model that could be executed, but rather a static representation with equipment states represented explicitly as nodes in the semantic network.

The purpose of this paper is to demonstrate that behavioural models that encompass both the control and manufacturing aspects of a PLC controlled manufacturing system can be effectively generated and that such models can then be used to develop applications which are of use in various stages of the system lifecycle. Our initial objective was to support activities in the maintenance phase (in particular fault diagnosis), but we have also developed applications which could support activities in the system design and system acceptance phases.

\section{MODEL CONSTRUCTION}

\subsection{The Pilot System}

The pilot system was a low-volume assembly line. It consists of 3 assembly stations (stations $10,20 \& 30$ ) linked by a transfer line. Two types of product are assembled on the line - Style A and Style B. The operations performed at each station are an alternating sequence of automatic and manual steps. Automatic operation at a station is initiated by an operator (or operators) depressing one or more sets of palm buttons. Those buttons remain depressed for the duration of that step. The activities performed during automatic operation typically involve the opening and closing of clamps. On completion of an automatic step (indicated by a lamp being illuminated on the station control panel), the operator removes his or her hands from the palm buttons and initiates a sequence of manual activities. This may involve the fixing of components to the sub-assembly, the loading of components, or perhaps removal of the sub-assembly from the station using external lifting equipment. 
When the assembly operations have been completed at a station, the operator needs to wait until assembly has been completed in the other stations. At that point, the transfer line is activated (by the operators at each station simultaneously depressing their palm buttons) and the cycle begins again with a new sub-assembly. Note that the number of stages in a cycle depends on the product being built.

\subsection{The Model}

A model of a PLC controlled manufacturing system, if it is to be used for fault diagnosis, will need to simulate the following activities:

a. PLC operation

A PLC executes the algorithm described in Figure 1:

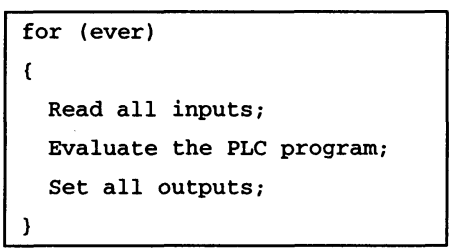

Figure 1 A simplification of the control loop typical of a PLC.

One loop is known as a scan. Our objective was not to emulate the detailed operation of the PLC so that accurate scan times can be determined, but rather to simulate its input / output behaviour. That is, having read all the PLC inputs, we need to determine what PLC outputs should be set by evaluating a representation of the PLC program.

b. Manufacturing System Operation

The PLC controls a manufacturing system. We can view the manufacturing system as consisting of 2 different entities:

1. Agents

2. Sensors

An agent is defined as a collection of electromechanical devices that can exist in one of several states. State selection is controlled by one or more PLC outputs. One or more events in the manufacturing process are associated with each agent state. As an example, agent SAV23 in the pilot system consists of two solenoids, an air valve, a piston and 3 clamps. The agent has two states designated SAV23A and SAV23B. In the first state, the clamps are open; in the second state, the clamps are closed. Sensors enable us to determine whether a particular manufac- 
turing event has occurred. Examples of sensors include limit switches, proximity switches and palm buttons. These entities typically interact as shown in Figure 2:

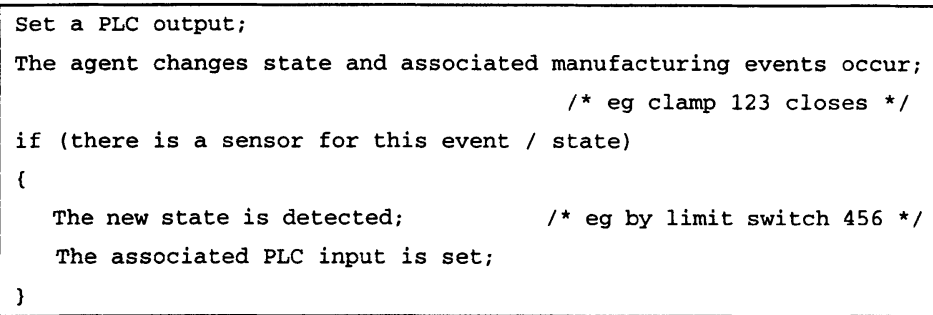

Figure 2 The sequence of events initiated by the setting of a PLC output.

Our primary interest is the causal relationships that exist between agent states and sensors. We are not concerned with the detailed operation of the individual agents but rather their state behaviour, as can be seen from the simulation trace illustrated in Figure 3:

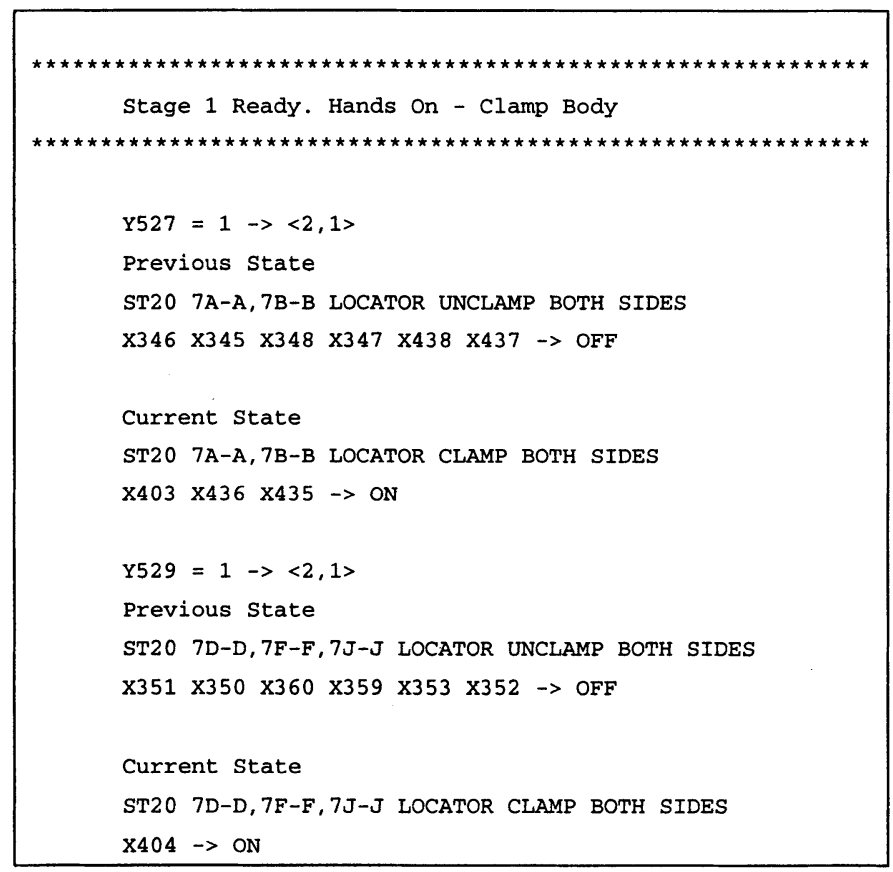




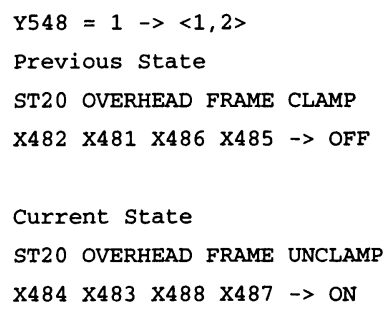

Figure 3 Simulation trace for part of stage 1 for station 20 .

Agents and sensors were implemented as finite state machines; agents change states when the appropriate PLC outputs are fired. When an agent undergoes a state transition, sensors associated with the previous state are deactivated, and those for the current state are activated. The PLC program was represented as a sequence of AND / OR graphs (one per rung); rung evaluation was viewed as a single-source shortest path problem for a graph with unweighted edges $^{1}$ (Weiss, 1993).

\section{APPLICATIONS}

Our primary objective has been to develop applications in support of the maintenance phase of the lifecycle presented in Section 1. However, in developing these applications, we have identified aspects of the system design and system acceptance phases which would benefit from model based tools. In a related activity (Jarvis and Jarvis, 1996) we have investigated the feasiibility of transforming PLC controlled manufacturing systems into distributed systems consisting of intelligent agents known as holons. One can view this as either a design or maintenance activity; we choose to view it as a maintenance activity.

\subsection{Maintenance}

Within the maintenance phase, we have developed tools to assist in system validation, fault diagnosis and system redesign. We also believe that documentation based on a system model would offer considerable advantages over existing approaches.

a. System Validation.

For each station, model validation proceeded in two phases. The outcome of Phase 1 was a model which, when started from the initial standby state, achieved cycle completion. Furthermore, the model could be run for more than one cycle (for this to happen, the state on cycle

1. Our objective is to determine whether a suitable path between the start node and the finish node exists, not to calculate the shortest such path. Consequently, we can use a shortest path algorithm, but stop as soon as a suitable path is found. 
completion needs to be the standby state). In Phase 2, a PLC memory image was captured at completion of each stage of the station cycle and compared with the state predicted by the simulation. Much to our surprise, not only did these comparisons highlight further modifications that needed to be made to our model, but they also identified several limit switches which had failed in such a way that the station was still completing its cycle (and consequently maintenance personnel were unaware of the problems). This was possible because of the way in which the system was designed. If one inspects the pneumatic circuit diagrams, one will see that in many cases, only one agent state (e.g. clamp open) is explicitly checked for with a limit switch. If the PLC program then only checks to see whether that event has happened, and if the limit switch associated with that event fails in the on position, PLC execution will continue as normal and the station will cycle normally. (Note however, that if the clamp fails to operate correctly for some reason, we have the potential for major damage to occur). A system for the detection of such faults (which we called benign) was developed and installed.

b. Fault Diagnosis

In constructing our diagnosis system, we assumed that

- there will be a single point of failure

- the point of failure will be a sensor (typically a limit switch)

The justification for the first assumption is that independent multiple faults which all contribute to a line stoppage are unlikely to occur. The second assumption can be relaxed if required; if it proves necessary we can induce faults in PLC outputs ${ }^{1}$. Note that this would allow us to diagnose multiple dependent faults; e.g. if a cylinder failed to advance, then all limit switches associated with all clamp actions associated with cylinder advancement would be in error.

The conceptual strategy that we used for diagnosis is presented in Figure 4.

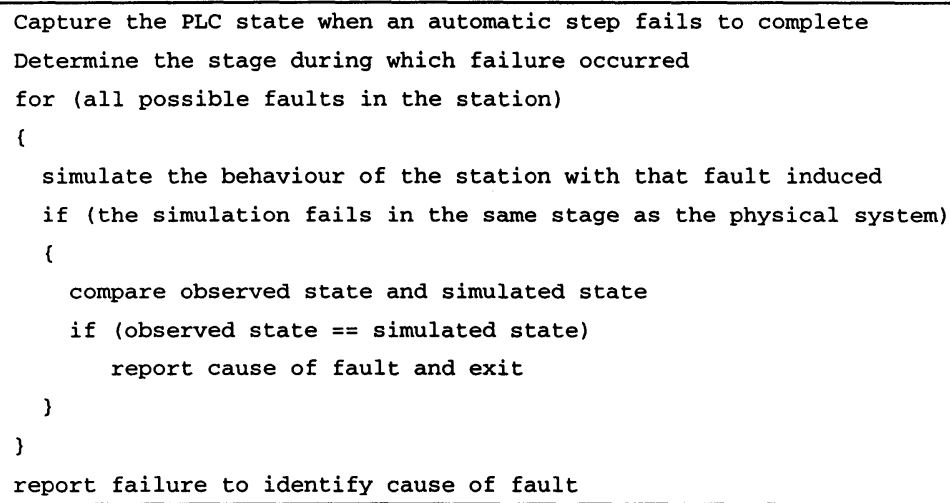

Figure 4 Conceptual strategy for model-based fault diagnosis.

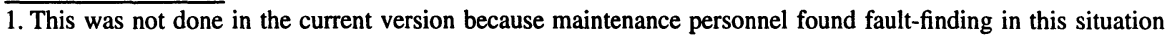
relatively straightforward. Their major difficulty is in finding faults associated with a single PLC input. 
In implementing this strategy, there are two major issues that need to be considered:

1. How do we determine the fault space?

2. How do we compare observed and simulated states?

The fault space for a particular station is the set of all possible faults that can occur. As we are dealing with a system which changes with time, a faulty limit switch will be characterised not only by its state (on or off) but also by the point in the cycle when the failure occurred. At first glance, the size of the fault space ${ }^{1}$ would suggest that the diagnostic strategy described above would be infeasible. However, as one would expect, the fault space can be pruned significantly. (Alternatively, degenerate fault states could be removed by inductive learning (Bratko et al, 1989)).

Comparison of observed and simulated fault states is not as complicated. The issue here is "What is the minimum amount of information that will enable us to identify a simulated state as being the same as the observed fault state?" We have chosen in the current implementation to do the comparison on the basis of selected inputs ${ }^{2}$. This has worked well in practice, although in general, a PLC state will be characterised by all its inputs, latched outputs and nonvolatile $\mathrm{C}$ memory locations.

The diagnostic system was validated by inducing faults during normal production and then attempting to ascertain the cause of the resultant stoppage by using the diagnostic system. The success rate during the validation and commissioning stages was $\sim 95 \%$. It takes $20-40$ seconds to perform a diagnosis; the system is currently running on a dedicated Sun IPC configured with $8 \mathrm{Mb}$ of RAM.

\section{c. System Redesign}

While PLCs have played a major role in the implementation of cost-effective automation, the style of programming that is required is not conducive to the development of modular control systems. It is worth noting that complex manufacturing systems (such as assembly stations) have a well defined hierarchical structure. However, it is difficult to construct PLC based control systems that reflect that structure. If one could do this, we believe that the verification and maintenance tasks for such systems would be significantly reduced. This problem is recognised by industry, and one solution that is emerging is to use separate PLCs for separate assembly functions. However, this solution is only being used for new systems and the problem of maintaining the integrity of the total manufacturing system still remains.

The concept of a holonic manufacturing system has recently emerged as a realistic candidate for the creation of the flexible and modular systems that the manufacturing sector will increasingly require (Deen, 1993; McFarlane et al, 1995). Holonic manufacturing systems will be constructed from entities known as holons, which will exhibit the dual characteristics of autonomous behaviour and the ability to function cooperatively. As such, they are similar to the multi-agent systems of distributed AI (Jennings, 1995). While holonic architectures offer the potential for systems which exhibit improved flexibility, reconfigurability and fault toler-

1. For Station 20 , there are approximately 17,000 points in the full fault space. This is the product of the number of input points ( 210 for Station 20) and the number of scans in the cycle ( 75 for Style A, 88 for Style B).

2. Due to production pressures, maintenance personnel were unable to prepare a system which was free of benign faults. Consequently, we chose to ignore those inputs which corresponded to benign faults. 
ance, most manufacturers are unlikely to embrace this new technology if its adoption requires them to discard their existing manufacturing and control systems. Manufacturers have an enormous investment in existing control technology, and what is required is a methodology which enables existing systems to be progressively evolved into holonic systems. We have demonstrated that the first step in this process, namely the creation of skeletal holonic subsystems which co-exist with conventional control systems is indeed feasible and that software can be developed which will enable the holonic subsystems to be automatically generated from a model of the existing manufacturing system (Jarvis and Jarvis, 1996). Note that we assume that holons have already been identified and correspond to the agents and sensors in our existing model.

In the holonic model, the only entities that will appear are holons. The control function is achieved by each holon maintaining knowledge of the states that it can adopt (e.g. clamp open, clamp closed) and the preconditions for its state transitions to occur. Preconditions are specified in terms of holon states. In the current model, notification of state transitions is achieved by a broadcast mechanism - when a holon changes state, all holons are notified. The key issue in the conversion of the PLC-based model to a holonic model thus becomes the extraction of preconditions for state transitions from the PLC-based model. The approach that we adopted (Jarvis and Jarvis, 1996) involved the following steps:

- transformation of the PLC-based model into an equivalent model with PLC inputs replaced by agent states.

- generation of a complete simulation trace for the agent state model

- extraction of the preconditions for every state transition from the simulation trace

- input the preconditions generated in the previous step into the holonic model and execute the model.

The approach has been tested successfully on the pilot system. An agent state model for the pilot system was generated, and preconditions were extracted for all state transitions. A holonic model of a subsystem of one of the stations was then constructed using the IDPS distributed programming environment ${ }^{1}$.

\section{d. Documentation and User Training.}

The model has been used by people unfamiliar with the the assembly line to gain an understanding of its operation. The usage of this capability has been limited because of the absence of an appropriate user interface. If an interface was provided which enabled maintenance engineers to interact with the model in an intuitive way (e.g. point and click on a ladder logic diagram), users could interact with the system in a "what if?" mode. Thus, user-directed diagnosis could be undertaken by inducing faults in the model and observing consequences. This mode of interaction would also be extremely useful in enabling engineers to gain an understanding of complex systems and to assess the impact of proposed changes to either the control system or the manufacturing system. Note that if our model was augmented with timing information for

1. IDPS is a programming environment which supports the development of prototype IDPS-OS applications. IDPS operates in a UNIX / TCP/IP environment. IDPS-OS (Seki et al, 1991) is a distributed, fault tolerant operating system which was implemented using reliable broadcasting between objects. 
the manufacturing events, the opportunity exists to explore the impact of control system modifications on cycle time.

As discussed earlier, the construction of a complete manufacturing model requires information to be extracted from several different sources. A consequence of this is that it is difficult to ensure that the information contained in all the sources is correct and consistent. Furthermore, we have highlighted through this exercise that the information which is normally maintained is insufficient to construct an operational model of a manufacturing system. It is therefore attractive to contemplate using the manufacturing model as the primary form of documentation. In this scenario, all information is held online and printed material (such as wiring diagrams) are generated from the online database. Consequently, changes can be better controlled, as we can now enforce a single entry point for system modifications.

\subsection{System Acceptance}

The system that we developed to detect benign faults, whilst deployed as a maintenance tool, could be deployed during system acceptance to ensure that the actual behaviour of the system corresponded to the "predicted" behaviour of the system.

\subsection{System Design}

During the course of the development and commissioning of the preceding applications, we developed a number of tools to identify potential design limitations in the manufacturing system and control system (e.g. limit switches which are only checked in one state, clamp positions which are not sensed, latches that don't latch, dependencies which could invalidate concurrent activities etc.). We recognise that if formal modelling techniques were used, such as Petri nets (Desrochers and Al-Jaar, 1995), process algebras (Milner, 1989) and temporal logics (Clarke et al, 1986), we could perform much more detailed and rigorous analyses of the system behaviour.

Petri nets have long been advocated as an alternative to ladder logic for the description of logic control systems. However, despite their advantages in terms of analysis and ability to represent concurrency, industrial perception was that they were too difficult to use (Desrochers and Al-Jaar, 1995). This led in 1977 to the development of GRAFCET, which is closely related to a subset of Petri nets called condition / event nets. However, no analysis can be done using GRAFCET, and place- and transition-invariants cannot be derived, which are essential for the verification of models. Also constraints on GRAFCET's evolution rules reduce its modelling power and applicability in applications which exhibit conflict, concurrency and asynchronous operation. Design frameworks based on Petri nets continue to be proposed (Ferrarini and Maffezzoni, 1993) but they have not as yet gained widespread acceptance. Frameworks based on finite state automata are beginning to emerge (Brandin and Charbonnier, 1994). This approach has the advantage that the concept of finite state machines is one which is well understood by maintenance engineers. With both approaches, a major challenge is to develop 
frameworks which can coexist with existing technology. In the short term, that may mean having design tools which generate PLC code.

The extent to which formal techniques can be used for the analysis of existing PLC controlled manufacturing systems is an issue that needs further investigation. In particular, the ability to effectively generate alternative representations for existing systems needs to be addressed. We have demonstrated through the creation of our holonic models that it is feasible to transform the existing system representation into a finite-state representation. Other transformations (to temporal logic (Moon, 1994) and to Sequential Function Charts (Falcione and Krogh, 1993)) have been reported. In both cases, the starting point is a user-generated control specification consisting of Boolean equations. Our experience with the maintenance procedures for large, complex systems suggests that if we want such files to accurately represent the control program, they must be generated automatically from the control program. Consequently, a key component of our modelling methodology is software that parses ladder logic listing files and produces AND / OR graphs. This software could be easily extended to generate Boolean equations and other representations as required.

Once an alternative representation has been constructed, one can then use analysis tools appropriate for that representation. A problem here is that for large systems, the state space is potentially enormous and analysis may become infeasible. A solution to this problem is to create models with higher levels of abstraction. Coloured Petri nets (Jensen, 1992) provide such a capability, as does the Circal process algebra (McCaskill and Milne, 1992). Unfortunately PLC programs do not lend themselves to abstraction because they indicate a functional, rather than a structural decomposition of a system. A much better starting point if abstraction is required is provided by the holonic models described above.

\section{CONCLUSION}

We have developed a model of an existing PLC-controlled assembly line which incorporates the behaviour of both the manufacturing system and the control system. This model was then used to develop a collection of model-based applications to support the maintenance and redesign of the assembly line. In the course of that work, we developed tools which would be of use in the design and acceptance phases of PLC-controlled manufacturing systems. We have also identified other applications which would benefit from the availability of behavioural models or from formal models.

\section{REFERENCES}

Asfahl, R.C. and Balagamwala, A. (1990), Simlog: a Prolog Based Simulator for Industrial Logic Control Systems. Computers and Industrial Engineering, 19, pp. 195-199.

Birrell, N.D. and Ould, M.A. (1994), A Practical Handbook for Software Development, Cambridge University Press. 
Brandin, B. and Charbonnier, F. (1994), The Supervisory Control of the Automated Manufacturing System of the AIP, In Proc. of the 4th. International Conference on Computer Integrated Manufacturing and Automation Technology, Troy New York, pp. 319-324.

Boullart, L. (1992), Using AI Formalisms in Programmable Logic Controllers. In Boullart, L., Krijgsman, A. and Vingerhoeds, R.A. (Eds.) Application of Artificial Intelligence in Process Control, Pergamon Press, pp. 96-113.

Clarke, E.M., Emerson, E.A. and Sistla, A.P. (1986), Automatic Verification of Finite-State Concurrent Systems Using Temporal Logic Specifications. ACM Transactions on Programming Languages and Systems, 8, pp. 244-263.

Cirocco, L.R., Jarvis, D.H., Jarvis, J.H. and Ryan A. (1995), Simulation of a PLC Controlled Assembly Station. Technical Report MTA 333, CSIRO Division of Manufacturing Technology, Adelaide, South Australia .

Day, W.B. and Rostosky, M.J. (1994), Diagnostic Expert Systems for PLC Controlled Manufacturing Equipment. International Journal of CIM, 7, pp. 116-122.

Deen, S.M. (1993), Cooperation Issues in Holonic Manufacturing Systems. In Yoshikawa, H. and Goosenaerts, J. (Eds.) Information Infrastructure Systems for Manufacturing (B-14), Elsevier Science, pp. 401-412.

Desrochers, A.A. and Al-Jaar, R.Y. (1995), Applications of Petri Nets in Manufacturing Systems: Modeling, Control and Performance Analysis. IEEE Press.

Falcione, A. and Krogh, B. (1993), Design Recovery for Relay Ladder Logic. IEEE Control Systems, 13, pp. 90-98.

Ferrarini, L. and Maffezzoni, C. (1993), Conceptual Framework for the Design of Logic Control. Intelligent Systems Engineering, 2, pp. 246-256

Jarvis, J.H. and Jarvis, D.H. (1996), A Strategy for Migration of a PLC-Controlled Manufacturing System to a Holonic Manufacturing System. In preparation.

Jennings, N.R. (1995), Controlling Cooperative Problem Solving in Industrial Multi-Agent Systems Using Joint Intentions. Artificial Intelligence, 75, pp. 195-240.

Jensen, K. (1992), Coloured Petri Nets. Volume 1: Basic Concepts. Springer-Verlag.

McFarlane, D., Marett, B., Elsley, G. \& Jarvis, D. (1995), Application of Holonic Methodologies to Problem Diagnosis in a Steel Rod Mill. In Proc. of the 25th. IEEE Conf. on Systems, Man and Cybernetics, Vancouver, pp. 940-945.

McCaskill, G.A. and MIlne, G.J. (1992), Hardware Description and Verification Using the Circal System, Research Report HDV-24-92, University of Strathclyde, Department of Computer Science, Glasgow, Scotland.

Milne, R., Nicol, C., Ghallab, L., Trave-Massuyes, L., Bousson, K., Dousson, C., Quevedo, J., Aguilar, J. and Guasch, A. (1994), TIGER: Real-Time Situation Assessment of Dynamic Systems, Intelligent Systems Engineering, 3, pp. 103-124.

Milner, R. (1989), Communication and Concurrency, Prentice Hall International Series in Computer Science.

Moon, I. (1994), Modeling Programmable Logic Controllers for Logic Verification. IEEE Control Systems, 14, pp. 53-59. 
Myers, D.R., Davis, J.F. and Hurley, C.E. (1990), A Knowledge Based Approach to Malfunction Diagnosis of Discrete Operations Involving Programmable Logic Controllers. In Proc. of the 1990 American Control Conference, San Diego, pp. 1974-1979.

Seki, T., Hasegawa, T., Okataku, Y. and Tamura, S. (1991), An Operating System for the Intellectual Distributed Processing System - An Object Oriented Approach Based on Broadcast Communication, Journal of Information Processing, 14, pp. 405-413.

Weiss, M.A. (1993), Data Structures and Algorithm Analysis in C. Benjamin / Cummings.

Wheeler, G. and Rossetti, V. (1993), Model Based Diagnosis in a PLC controlled Factory. In Proc. of Object Oriented Software, London.

\section{BIOGRAPHY}

Jacqueline Jarvis is a Lecturer in the School of Computer and Information Science at the Universty of South Australia. She holds a B.Sc. degree from the Flinders University of South Australia, and an MSc. in Software Development and Analysis from Heriot-Watt University. Her current research interest is model-based fault diagnosis.

Dennis Jarvis is a Principal Research Scientist at the CSIRO Division of Manufacturing Technology in Adelaide, South Australia. He holds a B.Sc. degree from the Flinders University of South Australia, and a Dip. Comp. Sci. and a Ph.D. degree from the University of Queensland. His current research interests are in cellular manufacturing, enterprise modelling and model-based fault diagnosis. He is a member of AAAI. 